\title{
Nonlinear Optimal Generalized Predictive Functional Control Applied to Quasi-LPV Model of Automotive Electronic Throttle
}

\author{
S. Alotaibi and M. Grimble*
}

\begin{abstract}
A Nonlinear Optimal Generalized Predictive Functional Control algorithm is presented for the control of quasi linear parameter varying state-space systems. A scalar automotive electronic throttle body is simulated to demonstrate typical results. The controller structure is specified in a restricted structure form including a set of pre-specified linear transfer-functions and a vector of gains that are found to minimize a GPC cost-index. This approach enables a range of classical controller structures to be used in the feedback loop such as extended PI, PID or of a more general transfer-function form. The controller is introduced along with a dynamic costweighting tuning future. A simulation is used to validate the performance of the restricted structure controller for regulation and tracking problems assessed against automotive performance standards.
\end{abstract}

\section{INTRODUCTION}

To satisfy automotive performance requirements and emissions legislation, it's important to achieve full electronic control of the engine. An Electronic Throttle Body (ETB) is a central module used to control the air intake and regulates the engine torque specially in Spark Ignition (SI) engines which require accurate control of both air and fuel. A typical ETB, as in Fig.1, consists of a throttle body with an electric motor attached to a butterfly valve through a set of gears and Throttle Position Sensors (TPS). The Electronic Throttle Control (ETC) provides the connection between the acceleration pedal and the ETB, using electrical signals as an alternative to a mechanical link. Engineering practice has revealed that the ETC system must provide a short settling time, small steadystate error, satisfaction of constraint and robustness against variations in ETB parameters (triggered by production deviations and the disturbance torque [1]). This type of rapid and accurate control obligation must also cope with the inherently nonlinearity and the time variability of the ETB mechanical structure [2].

Proportional Integral Derivative (PID) is often used to control an ETB because of its simplicity. However, in some cases, PID cannot achieve the desired performance in some operating conditions, especially when a low change in air flow rate is required and this is because of spring, friction nonlinearities and the disturbance torque effects. Thus, it would be reasonable to attempt to extend the capabilities of PID control expanding the controller to provide more design freedom. Several attempts on the control of ETB have been made, such as a PID controller with nonlinear compensator[1], [3], approximate model control strategy [4], [5], adaptive control strategy [6], constrained time optimal control strategy [7], selflearning PID control [8], fractional order fuzzy-PID [9].

\footnotetext{
* Authors are with the Department of Electronics and Electrical Engineering, University of Strathclyde, Glasgow, UK.
}

In this work, the nonlinear electronic throttle system was modeled as a quasi Linear Parameters Varying (qLPV) system. This is used to capture the ETB model nonlinearities. The valve angle and velocity were used as the scheduling variable and the disturbance torque (dependent on the air flow) and other causes, was treated as an exogenous input.

The paper is organized as follows: Modelling of ETB in qLPV form is outlined in Section II. The procedure to design the structure of the restricted structure controller for the ETB are discussed in section III. Section IV is dedicated to the discussion of simulation outcomes and Section V concludes this paper.

\section{EleCtronic Throttle Body MODELING}

The ETB DC motor dynamics in Fig. 2 was described in [10],[11] and can be represented by the following dynamics:

$$
V_{m}=i R+L \frac{d i}{d t}+E_{m}
$$

where $V_{m}, R, i$ and $L$ are motor voltage, resistance, armature current and inductance respectively. Here $E_{m}=K_{m} \dot{\theta}_{m}$ is the motor back Electro-Magnetic Field (EMF) voltage and $K_{m}$ is the EMF coefficient. The armature lag is neglected due to the small value of the armature inductance and also, the time constant of the motor has a smaller value in comparison to the sampling time $T_{s}$ [12]. The mechanical dynamics of the DC motor are given as:

$$
J_{m} \ddot{\theta}_{m}=T_{m}-K_{f m} \dot{\theta}_{m}-T_{g}
$$

where $J_{m}$ motor inertia, $K_{f m}$ motor friction coefficient, $\dot{\theta}_{m}$ motor angular velocity, $T_{m}$ motor armature torque and $T_{g}$ is the torque transmitted into the reduction gears. The torque generated by the armature $T_{m}=K_{a} i$ is linearly proportional to

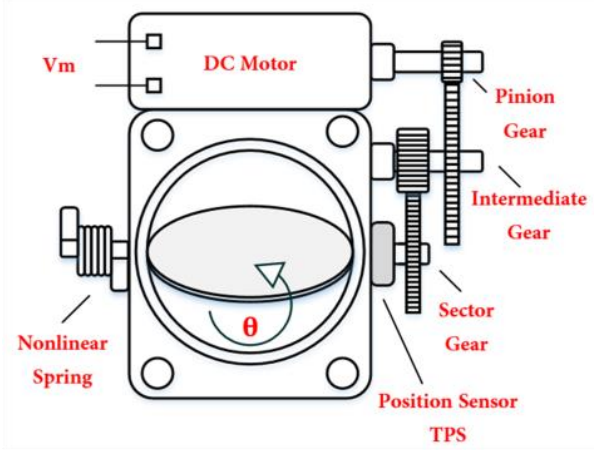

Figure 1. Typical ETB 


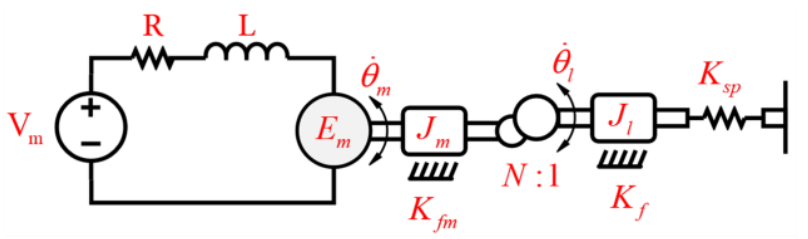

Figure 2. Lumped-element model of the ETB

the armature current and $K_{a}$ is the motor torque coefficient. The armature torque $T_{m}$ can be represented by:

$$
T_{m}=\frac{K_{a}}{R}\left(V_{m}-K_{m} \dot{\theta}_{m}\right)
$$

The load torque $T_{l}$ induced on the throttle valve shaft through the reduction gears is:

$$
T_{l}=N T_{g}
$$

where $N$ is the compound gear ratio. Finally, the dynamics of the throttle plate are described as:

$$
J_{l} \ddot{\theta}_{l}=T_{l}-\check{T}_{s p}-\check{T}_{f}-T_{d}
$$

where $\check{T}_{s p}, \check{T}_{f}$ and $T_{d}$ are dynamics resulting from the so-called Limp Home (LH) spring, friction and disturbances, respectively. The ETB has two inbuilt springs used as a failsafe mechanism. These springs force the valve plate to return to LH position somewhat above the shut location when there is no power applied to the ETB motor. This is to allow enough air to be provided to the engine when no control is presented. This torque is subject to whether throttle valve is in the LH position, forward or reverse direction. The movement of the valve plate is also limited by maximum and minimum angle limit. These restricted limits are treated as extremely stiff spring, preferably with infinite force as shown in Fig. 3. and can be expressed as in (6).

$$
\begin{gathered}
\check{T}_{s p}=K_{s p}\left(\theta_{l}-\theta_{0}\right)+T_{s p} \operatorname{sgn}\left(\theta_{l}-\theta_{0}\right) \\
T_{s p} \operatorname{sgn}\left(\theta_{l}-\theta_{0}\right)= \begin{cases}+T_{s p} & \theta_{0}<\theta_{l}<\theta_{\max } \\
-T_{s p} & \theta_{\min }<\theta_{l}<\theta_{0}\end{cases}
\end{gathered}
$$

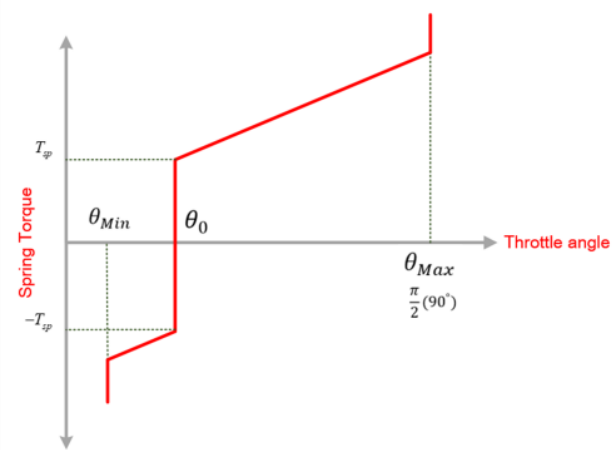

Figure 3. Return Spring Nonlinearity

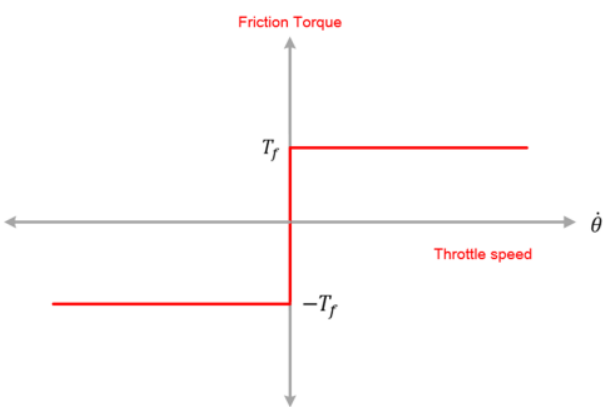

Figure 4. Coulomb Friction

where $T_{s p}$ spring preload torque, $K_{s p}$ stiffness coefficient of the spring, $\theta_{0}$ is the LH angle and sgn is the signum function. Another nonlinearity is due to friction, in which the force that is produced tends to oppose the motion of throttle plate such as linear viscous damping which has dependency on velocity and nonlinear coulomb friction demonstrated in Fig. 4. The total torque generated by friction can be demonstrated mathematically by (7):

$$
\begin{gathered}
\check{T}_{f}=K_{f} \dot{\theta}_{l}+T_{f} \operatorname{sgn}\left(\dot{\theta}_{l}\right) \\
T_{f} \operatorname{sgn}\left(\dot{\theta}_{l}\right)=\left\{\begin{array}{rr}
T_{f} & \dot{\theta}_{l}>0 \\
0 & \dot{\theta}_{l}=0 \\
-T_{f} & \dot{\theta}_{l}<0
\end{array}\right.
\end{gathered}
$$

where $T_{f}$ is the coulomb friction torque, $K_{f l}$ viscous friction coefficient and $\dot{\theta}_{l}$ is the valve angular velocity. Substituting (6) and (7) in (5) yields the following nonlinear dynamics of the throttle plate:

$$
\begin{gathered}
J_{l} \ddot{\theta}_{l}= \\
{\left[N T_{g}-\left[K_{s p}\left(\theta_{l}-\theta_{0}\right)+T_{s p} \operatorname{sgn}\left(\theta_{l}-\theta_{0}\right)\right]-\left[K_{f l} \dot{\theta}_{l}+T_{f} \operatorname{sgn}\left(\dot{\theta}_{l}\right)\right]-T_{d}\right]}
\end{gathered}
$$

Combining (2), (3) and (8) then, the differential equation representing the nonlinear model of the ETB become:

$$
\begin{gathered}
J_{t} \ddot{\theta}= \\
{\left[\frac{N K_{a} V_{m}}{R}-\left(K_{f t}+\frac{N^{2} K_{a} K_{m}}{R}\right) \dot{\theta}-T_{f} \operatorname{sgn}(\dot{\theta})-K_{s p} \theta-T_{s p} \operatorname{sgn}(\theta)-T_{d}\right]}
\end{gathered}
$$

where

$$
\begin{array}{cc}
J_{t}=J_{l}+N^{2} J_{m}, & K_{f t}=K_{f l}+N^{2} K_{f m} \\
\theta=\left(\theta_{l}-\theta_{0}\right), & \theta_{l}=N \theta_{m}
\end{array}
$$

During engine operation, there are various dynamic and disturbances that affect the tracking performance of the ETB, and these can be classified to measured varying parameter e.g. car battery voltage variation and unmeasured disturbance e.g. disturbance torque on the throttle plate induced by pressure variations due the air-flow dynamics of the engine therefore, the ETB qLPV state space model is given as:

$$
\begin{gathered}
\dot{x}=A_{0}(\rho) x+B_{0} u+D_{0} T_{d} \\
y=C_{0} x
\end{gathered}
$$


where

$$
x=\left[\begin{array}{l}
x_{1} \\
x_{2}
\end{array}\right]=\left[\begin{array}{l}
\theta \\
\dot{\theta}
\end{array}\right]
$$

where the elements of the state vector $x$ are the throttle angle and throttle angular velocity. The qLPV system depends upon parameters, the state vector $x$ and the control input $u$. The matrices $A$ and $B$ are usually nonlinear functions of the scheduling parameter $\rho$. qLPV systems is when any of the scheduling parmeters $\rho$ are a state of the system [13]. Finally, the components of (11) become:

$$
\begin{aligned}
& A_{0}=\left[\begin{array}{cc}
0 & 1 \\
-\frac{K_{s p}}{J_{t}}-\frac{T_{s p}}{J_{t}} \rho_{1} & -\frac{1}{J_{t}}\left(K_{f t}+\frac{N^{2} K_{a} K_{m}}{R}\right)-\frac{T_{f}}{J_{t}} \rho_{2}
\end{array}\right] \\
& B_{0}=\left[\begin{array}{c}
0 \\
\frac{N K_{a}}{J_{t} R}
\end{array}\right] \\
& D_{0}=\left[\begin{array}{c}
0 \\
-1
\end{array}\right] \\
& C_{0}=\left[\begin{array}{ll}
1 & 0
\end{array}\right] \\
& \rho_{1}=\frac{1}{\left|x_{1}\right|+\varepsilon}, \rho_{2}=\frac{1}{\left|x_{2}\right|+\varepsilon}
\end{aligned}
$$

where $\rho_{1}$ and $\rho_{2}$ are varying parameters calculated from measurement and estimation. The signum function $\operatorname{sgn}(x)$ in (9), can be approximated by $x /(|x|+\varepsilon)$, where $\varepsilon$ is a small positive scalar [14], used to guarantee a valid calculation when state $x$ goes to zero. However, the outcome of this approximation will be rounded to the nearest integer number in the online calculation and the effect of this approximation will be removed.

\section{NONLINEAR GPFC IN PID STRUCTURE}

The qLPV model shown in Fig. 5, denoted $W_{0}(\rho)$ is part of the augmented model which is assumed stabilizable and detectable. This augmented model can represent the discretetime state-space model described in (14) that results from directly discretizing (12). An alternative is to use a LPV identification algorithm to find this discretized model. It includes disturbance, control and error weighting subsystems. The approach of the augmented system method, created from the individual sub-systems, is defined in [15] and can be characterized in state-space form as in (15).

$$
\begin{aligned}
& x_{0}(t+1)=A_{0}(\rho) x_{0}(t)+B_{0}(\rho) u_{0}(t-k)+D_{0}(\rho) \zeta(t)+G_{0}(\rho) d_{0 d}(t) \\
& y(t)=d_{0}(t)+C_{0}(\rho) x_{0}(t)+E_{0}(\rho) u_{0}(t-k) \\
& z(t)=y(t)+v(t)
\end{aligned}
$$

\section{Augmented System State-Space Model}

$$
\begin{aligned}
& x(t+1)=A_{t} x(t)+B_{t} u_{0}(t-k)+D_{t} \xi(t)+d_{d}(t) \\
& y(t)=d(t)+C_{t} x(t)+E_{t} u_{0}(t-k) \\
& z(t)=y(t)+v(t) \\
& e_{p}(t)=d_{p}(t)+C_{p_{t}} x(t)+E_{p_{t}} u_{0}(t-k)
\end{aligned}
$$

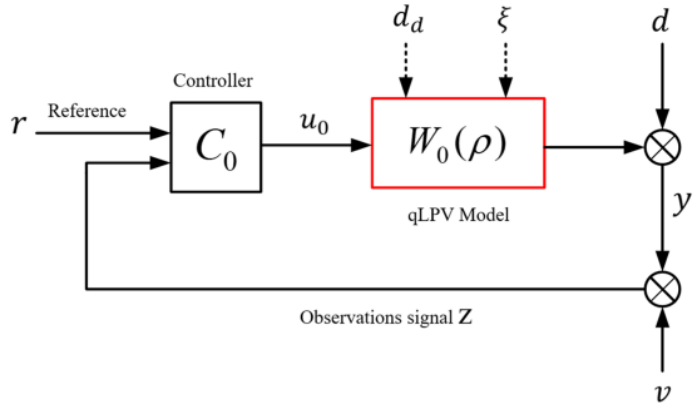

Figure 5. NGPFC for qLPV State Space Systems

where $y(t)$ the controlled output, $z(t)$ the measurement, $r(t)$ reference, $e_{p}(t)=P\left(z^{-1}\right)[r(t)-y(t)]=P\left(z^{-1}\right) e_{0}(t)$ is the weighted error and $P\left(z^{-1}\right)$ is the weighting operator. The reference is assumed known but can be represented by a stochastic model if needed. The measurement noise signal $v(t)$ and the stochastic component of the input disturbance $\zeta(t)$ are vector zero-mean, independent, Gaussian white noise signals. Noting, disturbances are broken down into their stochastic and deterministic components e.g. $d_{d}(t)$ planet input deterministic disturbance and $d(t)$ output deterministic disturbance.

The augmented system state space in (15) can be defined as follows:

$$
\begin{aligned}
& \overbrace{\left[\begin{array}{l}
x_{0}(t+1) \\
x_{d}(t+1) \\
x_{p}(t+1)
\end{array}\right]}^{x^{(t+1)}}=\overbrace{\left[\begin{array}{ccc}
A_{0}(\rho) & 0 & 0 \\
0 & A_{d} & 0 \\
-B_{p} C_{0}(\rho) & -B_{p} C_{d} & A_{p}
\end{array}\right]}^{A_{t}}\left[\begin{array}{c}
x_{0}(t) \\
x_{d}(t) \\
x_{p}(t)
\end{array}\right]+\left[\begin{array}{c}
B_{0}(\rho) \\
0 \\
-B_{p} E_{0}
\end{array}\right] u_{0}(t-k) \\
& +\underbrace{\left[\begin{array}{cc}
D_{0}(\rho) & 0 \\
0 & D_{d} \\
0 & 0
\end{array}\right]}_{D_{t}}\left[\begin{array}{c}
\zeta(t) \\
\omega(t)
\end{array}\right]+\underbrace{\left[\begin{array}{cc}
G_{0}(\rho) & 0 \\
0 & 0 \\
0 & B_{p}
\end{array}\right]\left[\begin{array}{c}
d_{0 d}(t) \\
(r(t)-d(t))
\end{array}\right]}_{d_{d}(t)}
\end{aligned}
$$

Similarly, the weighted error $e_{p}(t)$ can be defined as in (17)

$$
\begin{gathered}
e_{p}(t)=\overbrace{\left[E_{p}(r(t)-d(t))\right]}^{d_{p}(t)}+\overbrace{\left[\begin{array}{lll}
-E_{p} C_{0}(\rho) & -E_{p} C_{d} & C_{p}
\end{array}\right]\left[\begin{array}{l}
x_{0}(t) \\
x_{d}(t) \\
x_{p}(t)
\end{array}\right]}^{C_{p_{t}}}] \\
+\overbrace{-\left[-E_{p} E_{0}\right]}^{E_{p_{t}}} u_{0}(t-k)
\end{gathered}
$$

The prediction of the weighted error is essential in the control solution thus the $i$-steps prediction for the state can be gained by shifting the time by k-steps of the explicit transport-delay as in (18) and by repeated use of (16).

$$
\begin{aligned}
& x(t+i+k)=A_{t+k}^{i} x(t+k) \\
& +\sum_{j=1}^{i} A_{t+k+j}^{i-j}\left(B_{t+k+j-1} u_{0}(t+j-1)+D_{t+j-1} \xi(t+j+k-1)\right) \\
& +d_{d d}(t+k+i-1) \\
& \text { where } d_{d d}(t+k+i-1)=\sum_{j=1}^{i} A_{t+k+j}^{i-j} d_{d}(t+k+j-1)
\end{aligned}
$$


The future variations of control action are computed at each iteration, but future variations of parameters must be approximated. Collecting the deterministic disturbance signal components together in $d_{p d}(t+i+k)$ :

$$
d_{p d}(t+i+k)=d_{p}(t+i+k)+C_{p_{t+i+k}} d_{d d}(t+k+i-1)
$$

Then the weighted error $e_{p}(t)$ prediction can be obtained:

$$
\begin{aligned}
& e_{p}(t+i+k)=d_{p d}(t+i+k)+C_{p_{t+i+k}} A_{t}^{i} x(t+k) \\
& +\sum_{j=1}^{i} C_{p_{t+i+k}} A_{t+j}^{i-j}\left(B_{t+j-1} u_{0}(t+j-1)+D_{t+j-1} \xi(t+j+k-1)\right) \\
& +E_{p_{t+i+k}} u_{0}(t+i)
\end{aligned}
$$

The estimated weighted error signal may be composed in the following $N+1$ vector form

$$
\begin{aligned}
& \hat{E}_{P_{t+k, N}}=D_{P_{t+k, N}}+\overbrace{C_{P_{t+k, N}} A_{t+k, N}}^{C_{P N} A_{N}} \hat{x}(t+k \mid t)+\overbrace{\left(C_{P_{t+k, N}} B_{t+k, N}+E_{P_{t+k, N}}\right)}^{V_{P N}} U_{t, N}^{0} \\
& \hat{E}_{P_{t+k, N}}=\underbrace{0}_{\tilde{D}_{P_{t+k, N}}^{D_{P_{t+k, N}}}+C_{P_{N}} A_{N} \hat{x}(t+k \mid t)}+V_{P N} U_{t, N}^{0}
\end{aligned}
$$

where $\hat{x}(t+k \mid t)$ denotes a least squares state estimate from a Time-Varying Kalman Filter (TVKF). This is used with the qLPV model where the delays are accommodated on the input channels [16]. Time-varying matrices $A_{t}, B_{t}, C_{t}, E_{t}$ result in a time-varying error covariance matrix $P_{t}$ and hence a TVKF gain factor $K f_{t}$ derivation [17]. The Kalman estimation equation is summarized in the following:

$$
\begin{aligned}
& \text { (Predictor) } \\
& \hat{x}(t+1 \mid t)=A_{t} \hat{x}(t \mid t)+B_{t} u_{0}(t-k)+d_{d}(t) \\
& (\text { Corrector }) \\
& \hat{x}(t+1 \mid t+1)=\hat{x}(t+1 \mid t)+K f_{t+1}[z(t+1)-\hat{z}(t+1 \mid t)]
\end{aligned}
$$

where

$$
\hat{z}(t+1 \mid t)=d(t+1)+C_{t} \hat{x}(t+1 \mid t)+E_{t} u_{0}(t+1-k)
$$

The Kalman filter gain and Ricatti equations for a system with process and measurement noise covariance's $Q_{t}$ and $R_{t}$ :

$$
K f_{t+1}=P_{t+1 \mid t} C_{t+1}^{T}\left[C_{t+1} P_{t+1 \mid t} C_{t+1}^{T}+R_{t+1}\right]^{-1}
$$

$$
\begin{array}{ll}
\text { A priori covariance: } & P_{t+1 \mid t}=A_{t} P_{t \mid t} A_{t}^{T}+D_{t} Q_{t} D_{t}^{T} \\
\text { A posteriori covariance }: & P_{t+1 \mid t+1}=P_{t+1 \mid t}-K f_{t+1 \mid t} C_{t+1} P_{t+1 \mid t}
\end{array}
$$

The physical system to be controlled is assumed observable and controllable from the process noise inputs. Similarly, the $k$-steps ahead tracking error in (21), may consequently be described in the following vector form.

$$
\begin{aligned}
& E_{P_{t+k, N}}=D_{P_{t+k, N}}+C_{P N} A_{N} x(t+k) \\
& +V_{P N} U_{t, N}^{0}+C_{P N} D_{N} W_{t+k, N}
\end{aligned}
$$

Based on (22) and (27) the prediction error.

$$
\begin{aligned}
& \tilde{E}_{P_{t+k, N}}=E_{P_{t+k, N}}-\hat{E}_{P_{t+k, N}} \\
& =C_{P N} A_{N} \tilde{x}(t+k \mid t)+C_{P N} D_{N} W_{t+k, N}
\end{aligned}
$$

where the state estimation error in (29) is independent of the choice of control action

$$
\tilde{x}(t+k \mid t)=x(t+k)-\hat{x}(t+k \mid t)
$$

The optimal control solution using state-estimate prediction

$$
\begin{aligned}
& J=E\left\{\left(\hat{E}_{P_{t+k, N}}+\tilde{E}_{P_{t+k, N}}\right)^{T}\left(\hat{E}_{P_{t+k, N}}+\tilde{E}_{P_{t+k, N}}\right)+\right. \\
& \left.U_{t, N}^{0^{T}} \Lambda_{N}^{2} U_{t, N}^{0}+k_{c}^{T} \Lambda_{K}^{2} k_{c} \mid t\right\}
\end{aligned}
$$

The terms in cost-index (30) can be simplified, by noting the error optimal estimate is orthogonal to the estimation error.

$$
J=\hat{E}_{P_{t+k, N}}^{T} \hat{E}_{P_{t+k, N}}+U_{t, N}^{0^{T}} \Lambda_{N}^{2} U_{t, N}^{0}+k_{c}^{T} \Lambda_{K}^{2} k_{c}+J_{0}
$$

where

$$
\begin{gathered}
J_{0}=E\left\{\tilde{E}_{P_{t+k, N}^{T}}^{T} \tilde{E}_{P_{t+k, N}} \mid t\right\} \\
\Lambda_{N}^{2}=\operatorname{diag}\left\{\lambda_{0}^{2}, \lambda_{1}^{2}, \ldots, \lambda_{N}^{2}\right\} \\
\Lambda_{K}^{2}=\operatorname{diag}\left\{\rho_{0}^{2}, \rho_{1}^{2}, \ldots, \rho_{N}^{2}\right\}
\end{gathered}
$$

$E\{. \mid t\}$ represents the conditional expectation, conditioned on measurements up to time $t, \lambda_{j}$ represents a scalar control signal weighting factor and $\rho_{j}$ is the cost-weightings on the controller gains. The multi-step cost-function (31) may be extended as:

$$
\begin{aligned}
& J=\tilde{D}_{P_{t+k, N}^{T}}^{T} \tilde{D}_{P_{t+k, N}}+U_{t, N}^{0^{T}} V_{P N}^{T} \tilde{D}_{P_{t+k, N}} \\
& +\tilde{D}_{P_{t+k, N}^{T}}^{T} V_{P N} U_{t, N}^{0}+U_{t, N}^{0^{T}}\left(V_{P N}^{T} V_{P N}+\Lambda_{N}^{2}\right) U_{t, N}^{0} \\
& +k_{c}^{T} \Lambda_{K}^{2} k_{c}+J_{0}
\end{aligned}
$$

Before performing the optimization to calculate the optimal control, the controller structure will be introduced to have the desired PID form. This is different to model based unconstrained predictive control solutions, where the vector of future predicted controls is calculated and the control at time $t$ is implemented. The controller structure used here has a traditional cascade feedback form, where the role of various terms is obvious. So, for the scalar system e.g. ETB, the functions $f_{i}\left(z^{-1}\right)$ are chosen as in (34) using three linear dynamic functions $N_{e}$ to form a PID structure.

$$
u_{0}(t)=k_{c_{1}} \overbrace{f_{1}\left(z^{-1}\right)}^{1} e_{0}(t)+k_{c_{2}} \overbrace{f_{2}\left(z^{-1}\right)}^{k_{1-z^{-1}}} e_{0}(t)+k_{c_{3}} \overbrace{f_{3}\left(z^{-1}\right)}^{1-z^{-1}} e_{0}(t)
$$

and then the control signal could be realized as in (35)

$$
u_{0}(t)=\sum_{j=1}^{N_{e}} f_{i}\left(z^{-1}\right) k_{j}(t) e_{0}(t)
$$


To calculate the gain through the optimization, a suitable matrix representation is needed, therefore (35) can be rewritten as:

$$
\begin{gathered}
u_{0}(t)=e_{f_{s}}(t) k_{c_{s}} \\
=[\underbrace{\underbrace{f_{1}\left(z^{-1}\right) e_{0}(t)}_{P} \underbrace{f_{2}\left(z^{-1}\right) e_{0}(t)}_{I} \underbrace{e_{f_{s}}(t)}_{D}\left(z^{-1}\right) e_{0}(t)}_{P}]\left[\begin{array}{c}
k_{c_{s}} \\
k_{c_{1}} \\
k_{c_{2}} \\
k_{c_{N_{e}}}
\end{array}\right]
\end{gathered}
$$

where $s \in\left[1, N_{e}\right]$ and the PID time-varying gains can be collected as in (37)

$$
k_{c_{s}}(t)=\left[\begin{array}{c}
k_{c_{1}} \\
k_{c_{2}} \\
k_{c_{N_{e}}}
\end{array}\right]=\left[\begin{array}{c}
k_{p} \\
k_{i} \\
k_{d}
\end{array}\right]
$$

Using GPC strategy to calculate the gains in (37) is straightforward since the vector of future control action can be written as in (38)

$$
\begin{gathered}
U_{t, N}^{0}=\left[\begin{array}{c}
u_{0}(t) \\
u_{0}(t+1) \\
\vdots \\
u_{0}(t+N
\end{array}\right]=\overbrace{\left[\begin{array}{c}
e_{f_{s}}(t) \\
e_{f_{s}}(t+1) \\
\vdots \\
e_{f_{s}}(t+N)
\end{array}\right]}^{U_{f e}} k_{c_{s}}(t) \\
=U_{f e} k_{c_{s}}(t)
\end{gathered}
$$

where $U_{f e}$ is a matrix with $N+1$ rows and $N_{e}$ columns. A type of receding horizon strategy [18] is required, where the gain $k_{c}$ can be projected to be remain unchanged in the period $[0, N]$ and the calculated gains at time $t$ will be used to calculate at time $t$ optimal control action. At the next sample time instant the procedure can be repeated. By setting the gradient of the cost-function in (33) to zero [16], and substituting for the control input from expression (38) then the vector of future optimal controls can be calculated.

Constraints: If quadratic programming is used, constraints can be implemented on the controller gains value or even the rate of change of gains [19].

$$
k_{c_{s}}(t)=-\left(U_{f e}^{T}\left(V_{P N}^{T} V_{P N}+\Lambda_{N}^{2}\right) U_{f e}+\Lambda_{K}^{2}\right)^{-1} U_{f e}^{T} V_{P N}^{T} \tilde{D}_{P_{t+k, N}}
$$

Dynamic weightings were used to give more flexibility for engineers to tune the controller to minimize the feedback error. By selecting the error dynamic weighting terms performance can be adjusted i.e. whether controller action involves high or low gains. The error dynamical weighting can be designed using a frequency domain approach. The controller can be made to neglect unwanted high frequency dynamics or penalize the feedback error in a given frequency range. In this work, the dynamic weighting (40) was chosen as a PI and was discretized and converted to a state space model and augmented as in (16).
TABLE I. BOSCH ETB PARAMETERS

\begin{tabular}{c|c|c|c} 
Parameter & Value & Parameter & Value \\
\hline$N$ & 20.68 & $K_{s p}$ & $0.087 \mathrm{Nm} / \mathrm{rad}$ \\
$R$ & $1.15 \Omega$ & $K_{f t}$ & $0.0088 \mathrm{Nm} . \mathrm{s} / \mathrm{rad}$ \\
$J_{t}$ & $0.0021 \mathrm{~kg} \cdot \mathrm{m}^{2}$ & $T_{s p}$ & $0.396 \mathrm{Nm}$ \\
$K_{a}$ & $0.0185 \mathrm{Nm} / \mathrm{A}$ & $T_{f}$ & $0.284 \mathrm{Nm}$ \\
$K_{m}$ & $0.0185 \mathrm{~V} . \mathrm{s} / \mathrm{rad}$ & \\
\\
$e_{p}(t)=P\left(z^{-1}\right) e_{0}(t)=\left(K_{p_{p}}+\frac{K_{i_{p}}}{S}\right) e_{0}(t)$ \\
$x_{p}(t+1)=A_{p} x_{p}(t)+B_{p} e_{0}(t)$ \\
$e_{p}(t)=C_{p} x_{p}(t)+E_{p} e_{0}(t)$
\end{tabular}

\section{Simulation AND Performance Analysis}

The ETB parameters in Table I, were given in [20] and used in this simulation to assess the controller performance. The NGPFC-PID was first applied assuming known dynamics. The ETB controller reveals good tracking and regulation behaviors. The $1 \%$ settling time of $63 \mathrm{~ms}$ was observed for both small and big signals with maximum $1.7 \%$ overshoot. Similarly, a small steady state error observed at less than 0.05 degree, as shown in Fig. 6. The controller provides a performance beyond some automotive performance standards such as the S80 Volvo acceptable control responses [21].

The dynamic weighting is used to add an integral action to the controller and to also shape the performance. In this simulation phase, different dynamic weighting values was applied to show the flexibility in ETB performance tuning the calibration engineers may have.
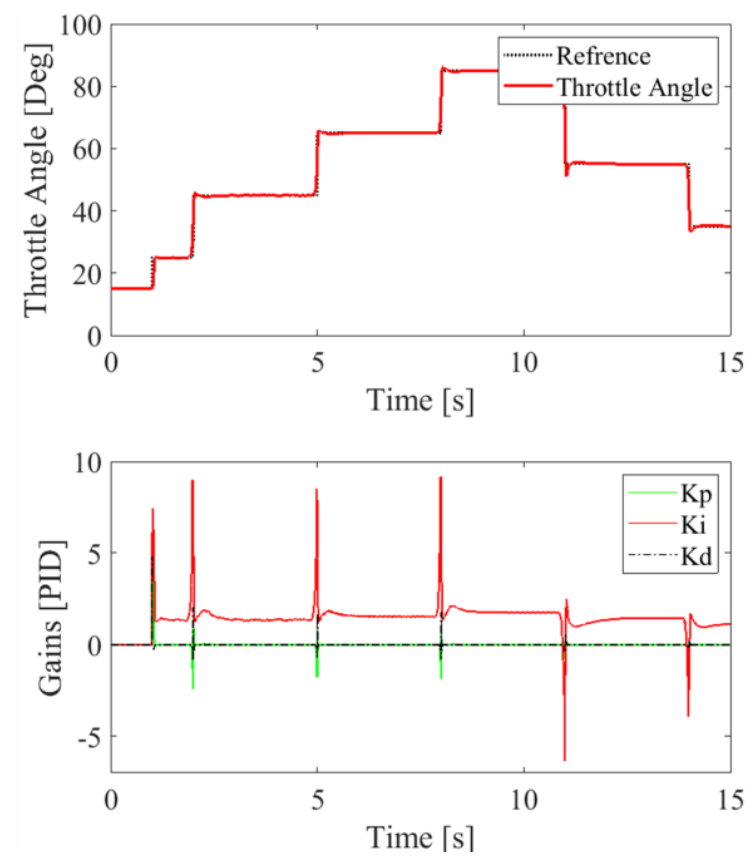

Figure 6. ETB NGPFC-PID Controller 

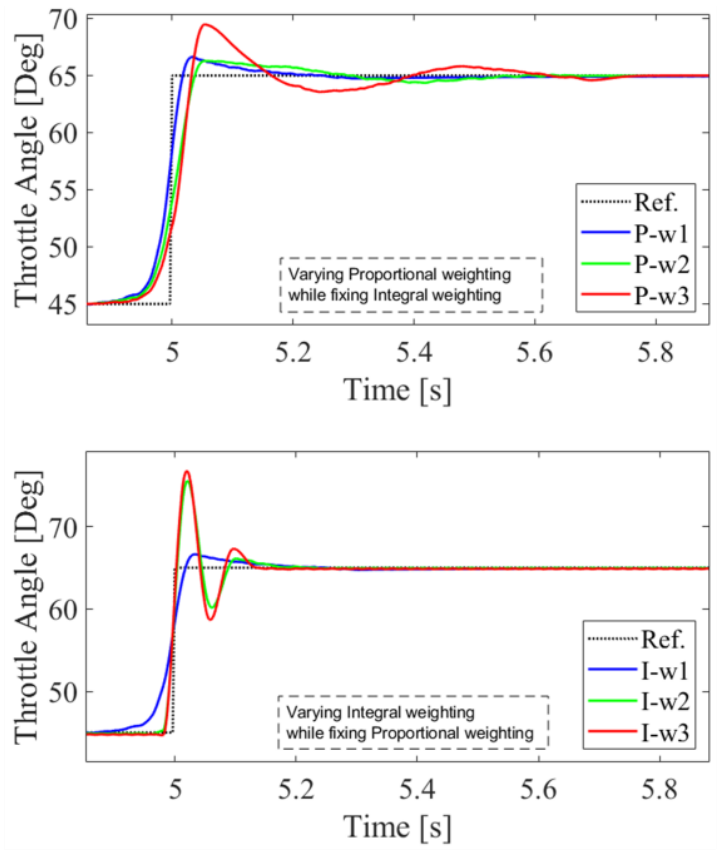

Figure 7. Dynamic Weightings Results

In Fig. 7 (Up), the proportional term of the dynamic weighting was kept constant against change in the integral term. The simulation reveals a decrease in steady-state error in response to the increase in the integral term and a steady state error of 0.001 Degree was achieved, but with slight overshot of $6 \%$.

In Fig. 6 (Down), the same procedure was followed fixing the value of the integral term whilst changing the proportional term value and the effect was measured against the rise time. A slight improvement of $15 \mathrm{~ms}$ was observed with an increased overshoot of $15 \%$ which could of course be suppressed by a reduction in the integral term.

A sports car performance demands are different from a car designed to minimise fuel. These results show how this fast servomechanism system could be calibrated. A new easy to use tuning feature for commissioning engineers is available to provide the required performance. Finally, the robustness of the restricted structure controller was evaluated against variability of the ETB parameters shown in Table I. The results demonstrate robustness against changes in ETB parameters.

\section{CONCLUSION}

In this paper, a non-linear GPFC controller has been designed based on ETB q-LPV model to control a nonlinear model of ETB. The controller has been set to have the structure of a PID design and was employed in a reference tracking problem where the optimized time varying controller gains are continuously updated. Using future prediction, the NGPCPF controller can adapt to the changes in reference trajectories, and changes in system operating regions due to friction and spring nonlinearities. The performance was assessed against some automotive performance standards and the NGPFC-PID controller results show improved performance, and its dynamic weighting tuning futures was used to improve the ETB transient responses.

\section{ACKNOWLEDGMENT}

The author S. Alotaibi would like to express his gratitude to Saudi cultural bureau UK for the sponsorship of his studies and to thank Dr Pawel Majecki, ISC Ltd. for his support on NPGMV toolbox software.

\section{REFERENCES}

[1] J. Deur, D. Pavkovic, N. Peric, and M. Jansz, "An electronic throttle control strategy including compensation of friction and limp-home effects," in IEEE International Electric Machines and Drives Conference, 2003. IEMDC'03., vol. 1, pp. 200-206.

[2] L. Eriksson and L. Nielsen, "Non-Linear Model-Based Throttle Control," 2000.

[3] D. Pavković, J. Deur, M. Jansz, and N. Perić, "Adaptive control of automotive electronic throttle," Control Eng. Pract., vol. 14, no. 2, pp. 121-136, Feb. 2006

[4] Xiaofang Yuan, Yaonan Wang, and Lianghong Wu, "SVM-Based Approximate Model Control for Electronic Throttle Valve," IEEE Trans. Veh. Technol., vol. 57, no. 5, pp. 2747-2756, Sep. 2008.

[5] Xiaofang Yuan and Yaonan Wang, "A Novel Electronic-ThrottleValve Controller Based on Approximate Model Method," IEEE Trans. Ind. Electron., vol. 56, no. 3, pp. 883-890, 2009.

[6] G. Panzani, M. Corno, and S. M. Savaresi, "On adaptive electronic throttle control for sport motorcycles," Control Eng. Pract., vol. 21, no. 1, pp. 42-53, Jan. 2013.

[7] M. Vasak, M. Baotic, I. Petrovic, and N. Peric, "Hybrid Theory-Based Time-Optimal Control of an Electronic Throttle," IEEE Trans. Ind. Electron., vol. 54, no. 3, pp. 1483-1494, Jun. 2007.

[8] X. Yuan and Y. Wang, "Neural networks based self-learning PID control of electronic throttle," Nonlinear Dyn., vol. 55, no. 4, pp. 385 393, Mar. 2009.

[9] W. Sheng and Y. Bao, "Fruit fly optimization algorithm based fractional order fuzzy-PID controller for electronic throttle," Nonlinear Dyn., vol. 73, no. 1-2, pp. 611-619, Jul. 2013.

[10] S. Zhang, J. J. Yang, and G. G. Zhu, "LPV gain-scheduling control of an electronic throttle with experimental validation," in 2014 American Control Conference, 2014, pp. 190-195.

[11] S. Zhang, J. J. Yang, and G. G. Zhu, "LPV Modeling and Mixed Constrained $\mathrm{H} 2 / \mathrm{H} \infty$ Control of an Electronic Throttle," IEEE/ASME Trans. Mechatronics, vol. 20, no. 5, pp. 2120-2132, Oct. 2015.

[12] M. Vasak, I. Petrovic, and N. Peric, "State estimation of an electronic throttle body," in IEEE International Conference on Industrial Technology, 2003, 2003, pp. 472-477.

[13] P. Gaspar, I. Szaszi, and J. Bokor, “Active Suspension Design using LPV Control,” IFAC Proc. Vol., vol. 37, no. 22, pp. 565-570, 2004.

[14] C. Edwards, S. Spurgeon, and S. Spurgeon, Sliding Mode Control. CRC Press, 1998.

[15] M. J. Grimble and P. Majecki, "Restricted Structure Predictive Control for Linear and Nonlinear Systems," Int. J. Control, pp. 1-61, 2018.

[16] M. J. Grimble and M. A. Johnson, Optimal control and stochastic estimation: theory and applications. Wiley, 1988.

[17] M. J. Grimble and P. Majecki, "NGMV Control Using Unstable StateDependent Multivariable Models." 2016.

[18] W. Kwon and A. Pearson, "A modified quadratic cost problem and feedback stabilization of a linear system," IEEE Trans. Automat. Contr., vol. 22, no. 5, pp. 838-842, Oct. 1977.

[19] M. J. Grimble and P. Majecki, "Restricted structure predictive control for linear and nonlinear systems," Int. J. Control, pp. 1-61, Oct. 2018.

[20] R. N. K. Loh, T. Pornthanomwong, J. S. Pyko, A. Lee, and M. N Karsiti, "Modeling, parameters identification, and control of an electronic throttle control (ETC) system," in International Conference on Intelligent and Advanced Systems, 2007, pp. 1029-1035.

[21] M. Svensson, "Robust design of an electronic throttle controller," Volvo Technological Development. Göteborg: Chalmers University of Technology, 1999. 